\title{
Docking Offset Between the Space Shuttle and the International Space Station and Resulting Impacts to the Transfer of Attitude Reference and Control
}

\author{
W. Jason Helms ${ }^{1}$ and Kara M. Pohlkamp ${ }^{2}$ \\ NASA Johnson Space Center, Houston, TX, 77058
}

\begin{abstract}
The Space Shuttle does not dock at an exact 90 degrees to the International Space Station (ISS) $x$-body axis. This offset from 90 degrees, along with error sources within their respective attitude knowledge, causes the two vehicles to never completely agree on their attitude, even though they operate as a single, mated stack while docked. The docking offset can be measured in flight when both vehicles have good attitude reference and is a critical component in calculations to transfer attitude reference from one vehicle to another. This paper will describe how the docking offset and attitude reference errors between both vehicles are measured and how this information would be used to recover Shuttle attitude reference from ISS in the event of multiple failures.

During STS-117, ISS on-board Guidance, Navigation and Control (GNC) computers began having problems and after several continuous restarts, the systems failed. The failure took the ability for ISS to maintain attitude knowledge. This paper will also demonstrate how with knowledge of the docking offset, the contingency procedure to recover Shuttle attitude reference from ISS was reversed in order to provide ISS an attitude reference from Shuttle.

Finally, this paper will show how knowledge of the docking offset can be used to speed up attitude control handovers from Shuttle to ISS momentum management. By taking into account the docking offset, Shuttle can be commanded to hold a more precise attitude which better agrees with the ISS commanded attitude such that start up transients with the ISS momentum management controllers are reduced. By reducing start-up transients, attitude control can be transferred from Shuttle to ISS without the use of ISS thrusters saving precious on-board propellant, crew time and minimizing loads placed upon the mated stack.
\end{abstract}

\footnotetext{
${ }^{1}$ GNC Flight Controller, Guidance and Controls Group, Space Shuttle Division, Mission Operations Directorate

${ }^{2}$ GNC Flight Controller, Guidance and Controls Group, Space Shuttle Division, Mission Operations Directorate
} 


\title{
Docking Offset Between the Space Shuttle and the International Space Station and Resulting Impacts to the Transfer of Attitude Reference and Control
}

\author{
W. Jason Helms ${ }^{1}$ and Kara M. Pohlkamp ${ }^{2}$ \\ NASA Johnson Space Center, Houston, TX, 77058
}

The Space Shuttle does not dock at an exact 90 degrees to the International Space Station (ISS) x-body axis. This offset from 90 degrees, along with error sources within their respective attitude knowledge, causes the two vehicles to never completely agree on their attitude, even though they operate as a single, mated stack while docked. The docking offset can be measured in flight when both vehicles have good attitude reference and is a critical component in calculations to transfer attitude reference from one vehicle to another. This paper will describe how the docking offset and attitude reference errors between both vehicles are measured and how this information would be used to recover Shuttle attitude reference from ISS in the event of multiple failures.

During STS-117, ISS on-board Guidance, Navigation and Control (GNC) computers began having problems and after several continuous restarts, the systems failed. The failure took the ability for ISS to maintain attitude knowledge. This paper will also demonstrate how with knowledge of the docking offset, the contingency procedure to recover Shuttle attitude reference from ISS was reversed in order to provide ISS an attitude reference from Shuttle.

Finally, this paper will show how knowledge of the docking offset can be used to speed up attitude control handovers from Shuttle to ISS momentum management. By taking into account the docking offset, Shuttle can be commanded to hold a more precise attitude which better agrees with the ISS commanded attitude reducing momentum management start up transients. By reducing start-up transients, attitude control can be transferred from Shuttle to ISS without the use of ISS thrusters saving precious on-board propellant, crew time and minimizing loads placed upon the mated stack.

\section{Nomenclature}

$\begin{array}{ll}\text { C\&C } & =\text { Command and Control } \\ C M G & =\text { Control Moment Gyroscope } \\ \text { COAS } & =\text { Crew Optical Alignment Sight } \\ \text { DAP } & =\text { Digital Auto Pilot } \\ \text { DDC } & =\text { Direct Deadband Collapse } \\ \text { FD } & =\text { Flight Day } \\ \text { FN } & =\text { Flight Night } \\ \text { GNC } & =\text { Guidance, Navigation and Control } \\ \text { GPS } & =\text { Global Positioning System } \\ \text { HUD } & =\text { Heads Up Display } \\ \text { IMU } & =\text { Inertial Measurement Unit } \\ \text { INTL } & =\text { Inertial } \\ \text { ISS } & =\text { International Space Station } \\ \text { LVLH } & =\text { Local Vertical/Local Horizontal } \\ M 50 & =\text { Mean of 1950 } \\ M C C & =\text { Mission Control Center } \\ M C S & =\text { Motion Control System }\end{array}$

${ }^{1}$ GNC Flight Controller, Guidance and Controls Group, Space Shuttle Division, Mission Operations Directorate

${ }^{2}$ GNC Flight Controller, Guidance and Controls Group, Space Shuttle Division, Mission Operations Directorate 


$\begin{array}{ll}\text { MET } & =\text { Mission Elapsed Time } \\ \text { MDM } & =\text { Multiplexer/Demultiplexer } \\ \text { ODS } & =\text { Orbiter Docking System } \\ \text { PRCS } & =\text { Primary Reaction Control System } \\ \text { REFSMMAT } & =\text { Reference to Stable Member Matrix } \\ \text { RGA } & =\text { Rate Gyro Assembly } \\ \text { STS } & =\text { Space Transportation System } \\ \text { TEA } & =\text { Torque Equilibrium Attitude } \\ \text { USTO } & =\text { U.S. Thrusters Only } \\ V R C S & =\text { Vernier Reaction Control System }\end{array}$

\section{Introduction}

Then the Space Shuttle docks to the International Space Station (ISS) the attitude commanded by the Orbiter's Digital Auto Pilot (DAP) is not the same as the attitude that is commanded by the ISS Motion Control System (MCS). By comparing the two systems' attitude knowledge over the course of many missions, noticeable differences have been found demonstrating it cannot be assumed the Orbiter docks at exactly 90 degrees to the ISS $\mathrm{x}$-axis (reference Figure 1) for many operations. This offset from 90 degrees, called the Orbiter Docking System (ODS) Offset, varies by several tenths of a degree throughout an orbit, and throughout a flight. Additionally, as the ISS construction continued over time, the offset angle has shifted with the increasing mass of ISS, the module locations, and other factors such as the use of the Space Shuttle airlock and the pressurization of the docking system.

From an operational point of view, it is important to know how the two attitude control systems are expected to compare for several reasons. First, when attitude control of the mated stack is transferred from Shuttle to certain ISS control modes, it can be critical to perform the handover in an attitude that matches as close to the ISS expected attitude as possible. Second, by knowing the comparison between the attitude knowledge of each docked spacecraft, it is possible to monitor the health of the attitude determination systems of the individual spacecraft and transfer attitude references between both vehicles for various malfunction scenarios.

\section{Overview of Shuttle and ISS Navigation and Control}

\section{A. Space Shuttle Navigation and Control Background}

On orbit, Space Shuttle attitude determination is derived from three Inertial Measurement Units (IMUs) which are mounted on a structural rigid platform called the "Navigation Base". During the prelaunch count, the IMU platforms are placed in specific attitudes with respect to the Local Vertical/Local Horizontal (LVLH) reference frame. The IMU platforms are held in these attitudes until approximately T-4 minutes, at which time the platforms are "released" to hold their designated inertially fixed attitudes. The transformation matrix between each IMU platform and the Mean of 1950 (M50) inertial reference frame is known as the Reference to Stable Member Matrix (REFSMMAT), and is stored in the Space Shuttle software. The Space Shuttle then determines its attitude based on the gimbal angles coming from the IMUs in conjunction with the "current" REFSMMAT. Note there are two REFSMMATS for each IMU, the "current" REFSMMAT defines the orientation of the IMU platform at the end of the previous alignment and the "desired" REFSMMAT defines the preferred IMU platform orientation at the next alignment.

Since the IMUs are mechanical devices, they drift over time due to friction. In order to support a deorbit at anytime, the IMU platforms are maintained to within 0.5 degrees. The IMU attitude error is monitored by comparing where navigation expected a star to be located in inertial space and where the Shuttle's star trackers actually track the star. The Shuttle has two star trackers named after their nearest Orbiter body axis (the -Y star tracker and the $-\mathrm{Z}$ star tracker). The star tracker line of sights are near 90 degrees apart such that when they both 
track a star the resulting star pair will have a separation near 90 degrees. These star pairs can then be used to calculate error in the IMU platform position. As the star separation angle moves from $90 \mathrm{deg}$, the accuracy of the calculation degrades slowly until the separation is $50 \mathrm{deg}$ (or $140 \mathrm{deg}$ ) and then rapidly beyond. Every few days or so in flight the IMU platforms are torqued back to their "desired” REFSMMAT position (aka. "aligned") using this information. Finally, a star must be within 1.4 degrees of where the onboard computers expected it in order for the star data to be accepted. Instead of using star data, an IMU can also be aligned to another IMU using its position as a reference.

For the Space Shuttle to perform attitude control on orbit, it compares the attitude and rate measurements from the IMUs to the desired attitude and rates entered by the crew into the DAP. If the errors in the attitude or rates exceeds the limits selected by the crew (deadbands), the DAP commands jets to fire in correction. The crew can select different DAP modes and deadband combinations depending upon the requirements for the current activity. The mode that is most commonly used is AUTO, which allows a number of different track attitudes, holds, and rotations. LVLH and Inertial (INTL) DAP options snap and hold the Orbiter's attitude to the appropriate reference frame when those modes are selected. The FREE drift DAP mode will inhibit all jet firings and not perform any attitude control.

The Orbiter has two different types of jets that can be used for control: Primary Reaction Control System thrusters (PRCS) and Vernier Reaction Control System thrusters (VRCS). There are 38 PRCS jets each providing 870 pounds of vacuum thrust. The PRCS jets can be operated with two different software schemes: "PRI" mode that uses a simple look up table to determine which jet(s) to fire for a specific body axis error, and "ALT" mode that determines the "optimal" jet(s) to fire based on additional considerations including the number of jets allowed to fire at once and set delay times between firings. As such, ALT is more propellant efficient and induces smaller loads while mated to ISS. PRI is not certified for mated operations. There are six vernier jets that provide 24 pounds of vacuum thrust. Verniers are only used on-orbit and provide much finer control. Since they induce smaller loads, they are the preferred method of Orbiter control for the mated stack but are also zero-fault tolerant (all six jets are required for mated control).

\section{B. ISS Navigation and Control Background}

The US segment ISS attitude knowledge is determined by a Kalman filter selection based from differential GPS with RGA rate propagation between updates. The attitude reference can be updated with data from the Russian segment and/or ground uplinks as required. The Russian state is determined using a strapped down inertial navigation system called БИНС (pronounced "beans"). When the Russian state is updated it is called a "БИНС correction" which is typically performed with the Russian star trackers.

Attitude control is nominally maintained through the use of four Control Moment Gyroscopes (CMGs) which reduce the need to use propellant (a precious consumable) and allows the ISS to maintain a "microgravity" mode for scientific experiments. For orbital adjusts and large maneuvers, ISS utilizes 16 Russian thrusters as well as thrusters from non-Shuttle visiting vehicles (Soyuz, Progress, HTV, ATV, etc). These thrusters can be commanded in a "Russian Thruster" mode where the Russian segment is in command, or in a US Thruster Only (USTO) mode where the US segment commands the Russian Thrusters. Thrusters are also utilized to dump CMG momentum called a "desaturation." Nominally, when Orbiter is docked to ISS the mated stack flies in a torque equilibrium attitude (TEA) which has minimized disturbance torques to allow for momentum management control and also minimizes propellant consumption when thrusters are utilized.

The US segment GNC system is controlled by the GNC Multiplexer/Demultiplexer (MDM) which is controlled by the higher tier Command \& Control (C\&C) MDM. A similar tiered computer structure exists on the Russian side with the TVM and TsVM computers being roughly equivalent to the C\&C and G\&C MDMs of the US segment respectively.

\section{Calculation of the ODS Offset}

The ODS offset is calculated by comparing time homogenous inertial attitudes of the Orbiter and ISS. The offset is calculated as the quaternion, $\mathrm{Q}_{\mathrm{B}_{\mathrm{I}} \mathrm{ISS}}$, representing the rotation from the Orbiter body frame to the ISS body frame. The inertial attitude of the Orbiter downlisted in telemetry as the quaternion, $\mathrm{Q}_{\mathrm{B}_{-} \mathrm{I}}$, represents the rotation from the Orbiter body axes to the M50 reference Inertial frame as determined by the onboard selected IMU. Q Q downlisted once a second, and is captured in the same time as the quaternion $\mathrm{Q}_{\mathrm{J} 2 \mathrm{~K} \_\mathrm{ISS}}$, which is the rotation from the 
$J 2 K$ inertial reference frame to the ISS body axes downlisted in the ISS data stream. Since M50 and J2K are two different inertial reference frames, a conversion must be made using the rotation between M50 and J2K, $\mathrm{Q}_{\mathrm{M} 50 \_ \text {J2K, }}$ which is a constant angle of rotation of 0.6984 deg. Q $_{\text {B_IsS }}$ is calculated (as shown in Equation 1).

$$
\begin{aligned}
\mathrm{Q}_{\mathrm{B}_{-} \mathrm{ISS}}=\mathrm{Q}_{\mathrm{B}_{-} \mathrm{I}} \cdot \mathrm{Q}_{\mathrm{M} 50 \_\mathrm{J} 2 \mathrm{~K}} \cdot \mathrm{Q}_{\mathrm{J} 2 \mathrm{~K} \_\mathrm{ISS}} \\
\text { where } \mathrm{Q}_{\mathrm{M} 50 \_\mathrm{J} 2 \mathrm{~K}}=\left[\begin{array}{c}
0.9999814268044 \\
-0.0000000021678 \\
0.0024295468899 \\
-0.005589574961
\end{array}\right]
\end{aligned}
$$

Solving for $\mathrm{Q}_{\mathrm{B}_{\_} I S S}$ when $\mathrm{Q}_{\mathrm{B}_{\_} \mathrm{I}}$ and $\mathrm{Q}_{\text {J2K_IISS }}$ are known is dubbed performing an ODS “calibration.” Since the ODS offset is calculated by comparing the inertial attitude reference of both vehicles, it is important to perform this calculation when each vehicle's attitude reference is as accurate as possible. While the Orbiter is docked to ISS the $\mathrm{Z}$ star tracker line of sight is blocked by ISS structure and the -Y star tracker field of view is often partially blocked by ISS solar arrays and robotic activities. Also, very few (if any) mated maneuvers occur during the docked timeframe depending upon mission objectives. Due to these constraints, the star pairs that the $-Y$ star tracker can track by itself while docked have very small separation angles ( 30 degrees for good pairs). Thus the Orbiter has the most accurate attitude reference during the docked timeframe immediately after docking. Since the ISS also ensures it has a good attitude state in support of docking, the most accurate calibration is measured after the maneuver to the docked TEA attitude. This allows disturbances from the docking mechanism and maneuvers time to dissipate, but provides the most accurate attitudes available.

In order to determine a good time to perform the ODS offset calculation, the Orbiter to ISS structural flexure and differences between the two attitude reference systems are monitored in MCC along with the time tags of the

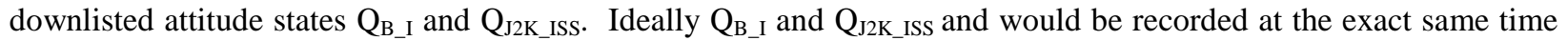
but time differences are introduced by the unique telemetry processing of each vehicle. Shortly after hard mating and an "initial" time difference is recorded as the difference in the time tags of the downlisted $Q_{B_{-}}$from the time tag of $\mathrm{Q}_{\mathrm{J} 2 \mathrm{~K} \_\mathrm{ISS}}$ in seconds. For each subsequent cycle, the difference in the time tags from the initial time difference is plotted to provide a means to monitor the relative time tag difference. An example of this is shown in Figure 2 during a 4.5 hour example from STS-135. Missing data in the plot corresponds to nominal loss of data from the vehicles to MCC.

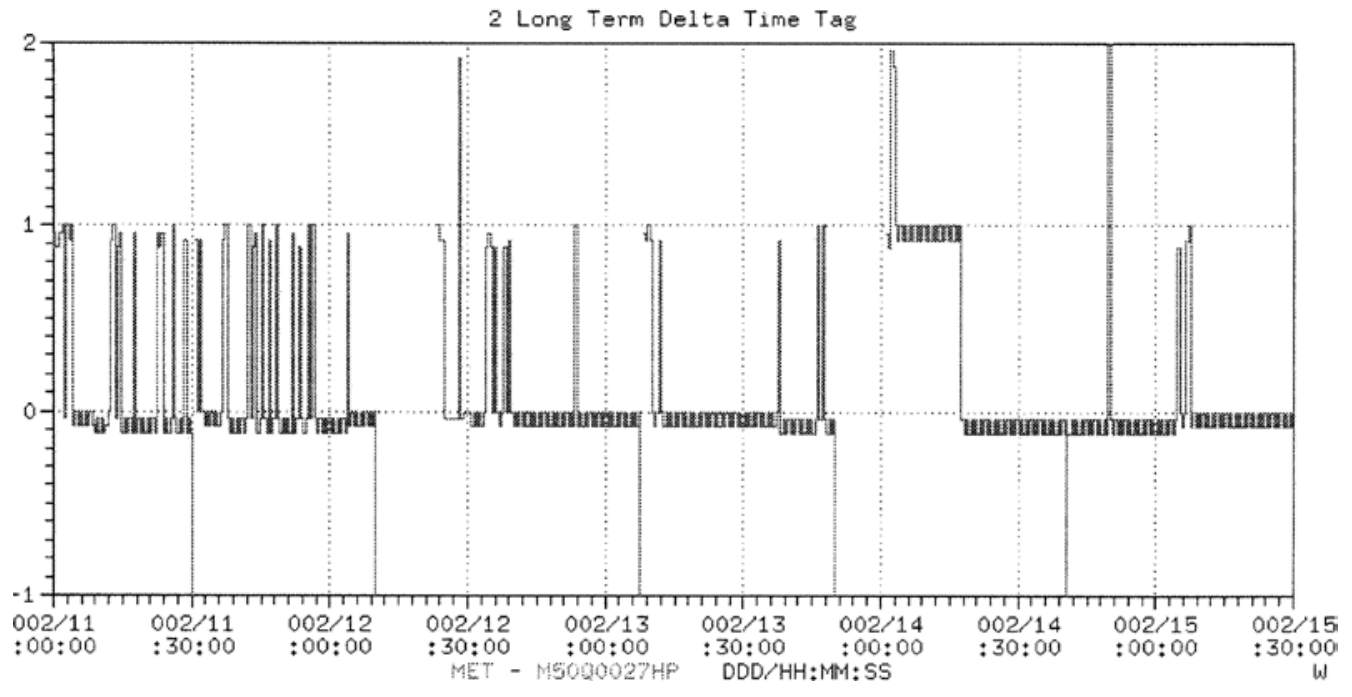

Figure 2. Delta Time Plot Example from STS-135. 


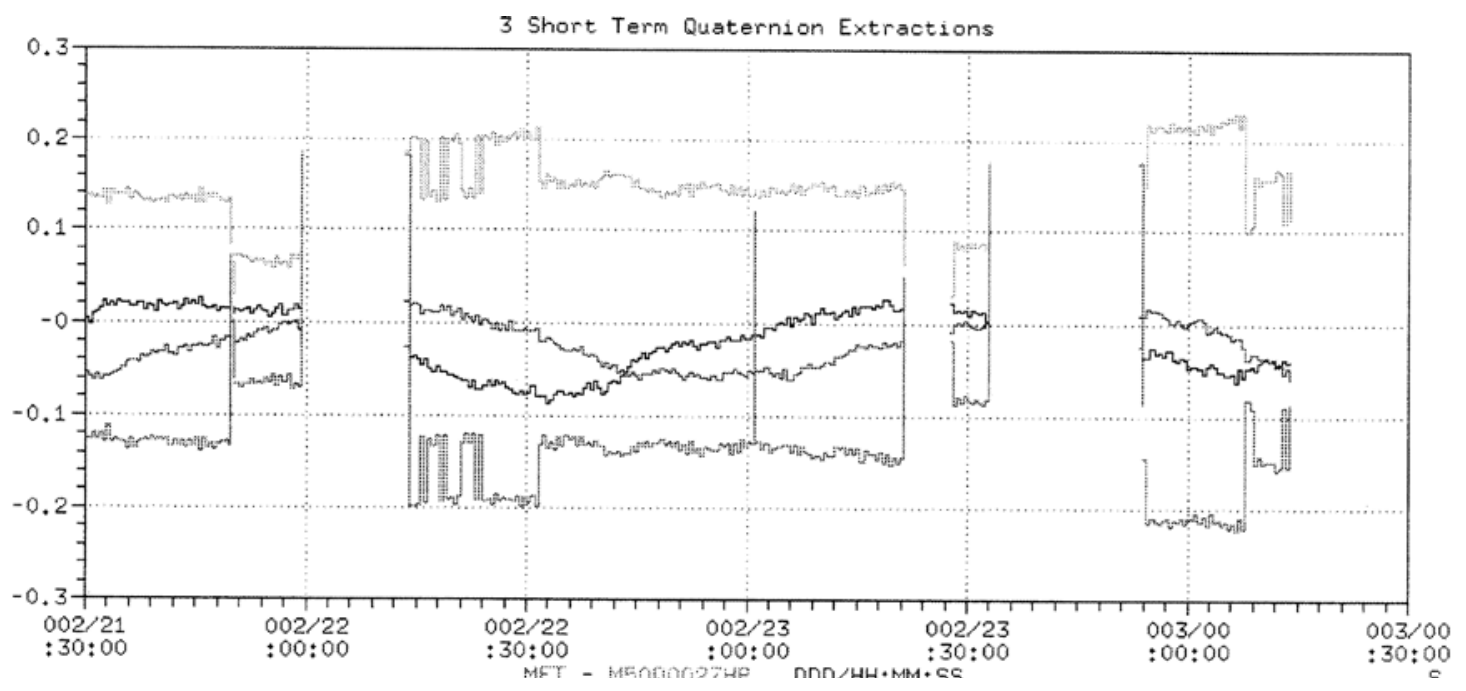

Figure 2. Quaternion Extraction Plot Example from STS-135.

To monitor the structural flexing a similar strategy is used. At the same time the time tag difference is initialized, a $\mathrm{Q}_{\text {B_Iss }}$ is also calculated as an "initial” value. For each subsequent cycle the difference of the current cycle's $\mathrm{Q}_{\text {В_Iss }}$ with the initial value is computed and the eigen angle extraction is plotted. An example of this is shown in Figure 3 during a 3 hour example from STS-135 where the top line is an RSS of the Eigen angle and the bottom three lines are the individual angle components (Pitch, Yaw and Roll). Structural oscillations can then be monitored with each cycle corresponding to a revolution (aka rev) of the mated stack around the Earth.

Since the quality of the initial snap shots is unknown, these plots are used to monitor patterns and not quantative differences. After watching the behavior for several revs, a final $\mathrm{Q}_{\mathrm{B}_{\mathrm{I}} \mathrm{ISS}}$ is recorded when the delta time and eigen angle extraction differences are as near as possible to their middle of their respective ranges. This quaternion is documented and represents the best possible calculation of the actual ODS offset and is dubbed the "Calibration".

In MCC, two instances of the delta time and eigen angle extraction difference comps are maintained. This allows one of the computations to be "reinitialized" every so often while still maintaining a longer-term history of these parameters. These comps are called the "long term" and "short term" computations.

\section{ODS Offset History}

The ODS offset has been calculated for every Shuttle docking to ISS since STS-114 which docked July 28, 2005. Over time, it became apparent that a number of factors affect the ODS offset. Figure 4 shows the Euler angle components of the ODS offset for 6 hours (4 orbits) taken during STS-120, with OV-103 Discovery. This data was calculated during crew sleep on Flight Night (FN) 4 which ensures quiescent operations (no activities occurred that would change mass properties or cause large structural perturbations such as waste water dumps, attitude control system jet firings, and most significantly EVA or robotics activities). This data was also coordinated with the ISS flight controllers to ensure that the attitude knowledge downlisted by ISS was accurate, in this case to approximately 0.2 degrees. During the same time, the Space Shuttle IMUs were accurate to 0.04 deg as measured by star data using the -Y star tracker. The mated stack was in a LVLH TEA called -XLV -ZVV since the Orbiter -X axis pointed towards the earth (Local Vertical) and the $-Z$ axis pointed into the velocity vector. In other words, the Orbiter was nose up with the payload bay into the wind. 
Figure 4 shows oscillations can be seen in all three axes with a frequency corresponding to the orbital period and are most likely caused by attitude sensitivities within the IMUs.

The ODS offset euler angle extractions shown in Figure 5 were recorded during STS-118 when OV-105 Endeavour was docked to ISS. STS-118 was the last flight of the Spacehab module that connects to the docking mechanism with a pressurized tunnel that extends into the payload bay providing additional room for scientific experiments. The presence of Spacehab causes a different structural configuration between the Shuttle and ISS which effects the ODS offset pitch angle. Data from STS-120 shows the average pitch calibration is -91.4 deg. where the STS118 pitch has an average of -90.2 deg. This contrast is consistent for flights with and without Spacehab. Additionally, no significant change is seen in the roll or yaw components between flights with and without a Spacehab module.

When the ODS offset is graphed over a longer time period the effects of attitude updates from both Shuttle and ISS are apparent. When the Orbiter IMUs are aligned a step is observed in the ODS offset as shown in Figure 6. This plot shows the ODS offset recorded in three minute increments. The IMU alignment was performed at 144.05 hours into the mission and is evident by the data point at MET 144.08 hours.

Similarly, attitude updates on ISS cause step jumps in the ODS offset calculation. Figure 7 shows the ODS offset pitch angle from STS-120 over nearly three days. At MET hour 225.3, ISS reset their attitude filter which caused a step in the ODS offset calculation. Similarly, the step at MET hour 244.5 was caused by ISS swapping their attitude filter from string 2 to 1 and the step at hour 245.7 was caused by а БИНС correction.
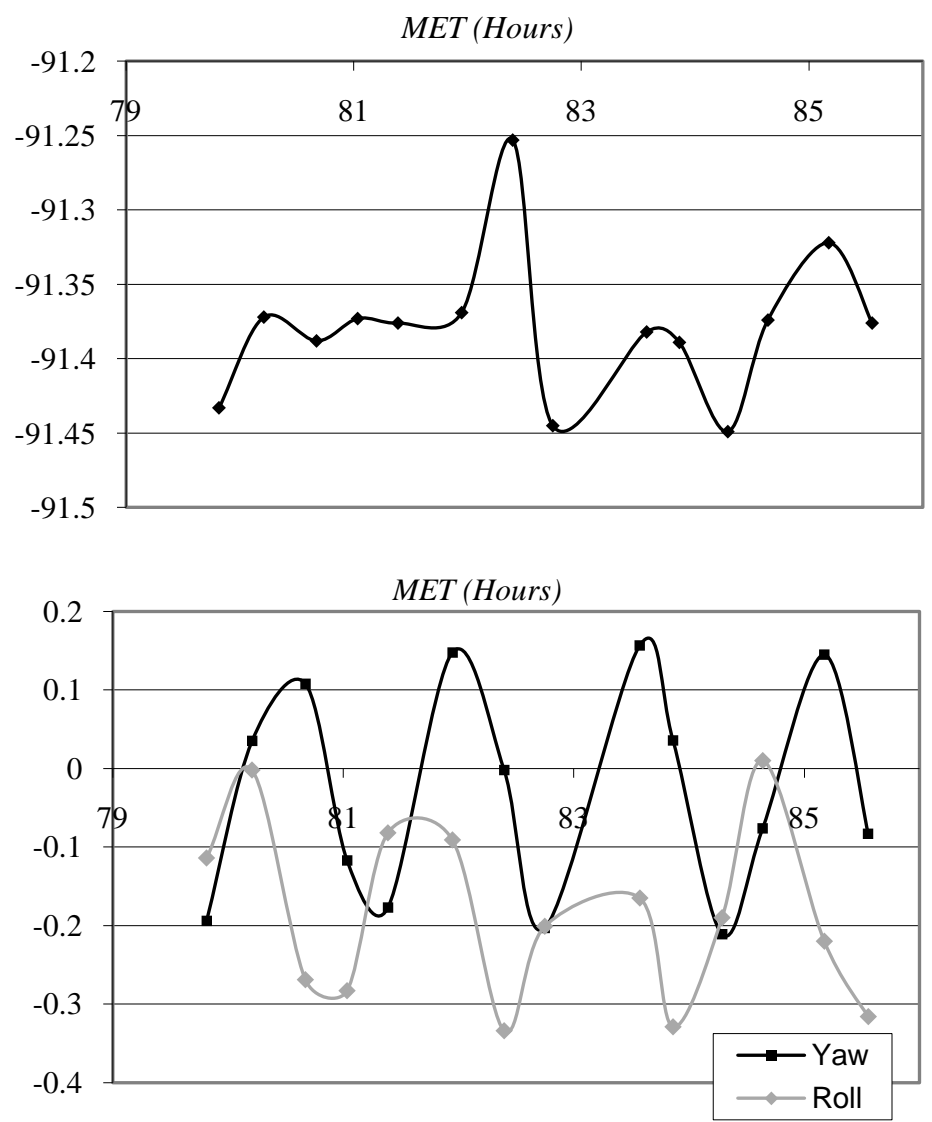

Figure 4. STS-120 ODS offset euler angle extractions expressed in Orbiter body axes over a time period of about 6 hours.

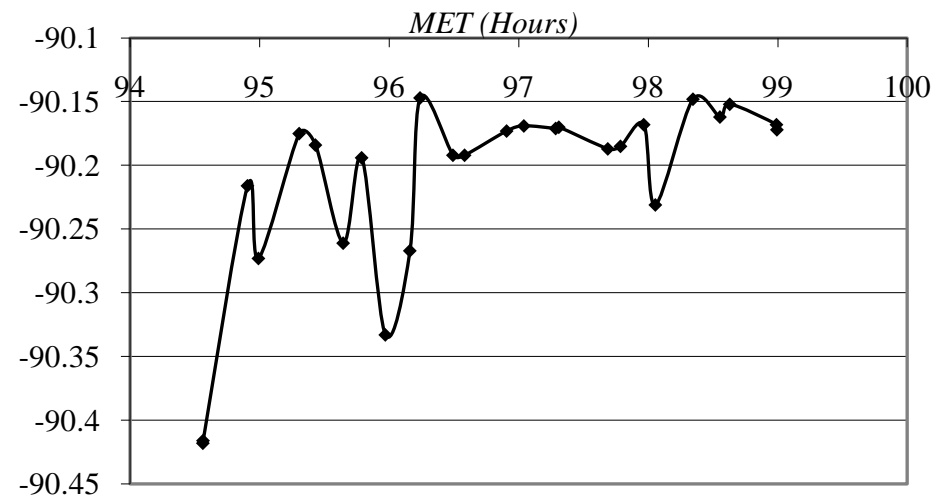

Figure 5. STS-118 ODS offset pitch angle expressed in the Orbiter body axis. 


\section{Orbiter Attitude Reference Recovery from ISS}

Calculation of the ODS offset was initially started to protect for the multiplefailure deep case of loss of attitude reference on Shuttle. Once a "calibration" ODS offset is recorded, this information can be used to recover Shuttle attitude knowledge using ISS as a reference. This contingency procedure has never been required or used during a real mission.

In 2007, a software update was made to Orbiter flight software to allow an IMU "matrix alignment." For this alignment the IMU being aligned is not torqued (the platform is not moved at all). Instead, an accurate REFSMMAT is calculated from the IMU being used as a reference such that it accurately describes the current position of the IMU platform being aligned. Thus the alignment occurs mathematically and not physically. This software update greatly simplified the procedure to recover attitude reference from ISS. Both versions of this procedure are described below.

\section{A. Without IMU Matrix Aligns}

Once Shuttle attitude reference has been lost, the short-term delta time and eigen angle extraction computations are reinitialized. These computations became invalid with the loss of the reference attitude, and restarting the short-term computation allows the midpoint of structural oscillations to be found using a new if erroneous attitude. The long-term computation is not restarted. Since the comp will become valid once attitude reference is regained, this computation is the fastest method of verification that attitude reference has be successfully recovered.

When the computation of the short-term relative attitude deltas indicates the three axes are as near as possible to the center of their ranges (structural flex and perturbations are minimized), the $\mathrm{Q}_{\mathrm{R}_{-} \mathrm{Sm}}$ quaternion associated with each IMU as well as the $\mathrm{Q}_{\mathrm{J} 2 \mathrm{~K} \_ \text {_Is }}$ quaternion is recorded. $\mathrm{Q}_{\mathrm{R} \_\mathrm{SM}}$ is the quaternion equivalent of each IMU's current gimbal angles (the rotation from the physical IMU casing to the IMU's platform). If attitude reference recovery must be accomplished quickly, these values can be recorded without regard to the short-term computation but the recovery error will be larger. If the recovery error ends up being greater than $1.4 \mathrm{deg}$, the star trackers will not be able to track stars and the entire procedure would need to be repeated.

From $\mathrm{Q}_{\mathrm{R}_{-} \mathrm{SM}}$, the conjugate of $\mathrm{Q}_{\mathrm{I}_{\mathrm{B}}}$, $\mathrm{Q}_{\mathrm{B} \_\mathrm{I}} \mathrm{can}$ be calculated. However, this quaternion now represents an erroneous rotation from the inertial frame to the Orbiter since attitude reference has been lost:

$$
\mathrm{Q}_{\mathrm{B}_{-} \mathrm{I}}=Q_{B_{-} N B} \cdot Q_{N B_{-} R} \cdot Q_{R_{-} S M} \cdot Q_{C_{-} M 50}
$$

Where: 
$\mathrm{Q}_{\mathrm{B} \_\mathrm{NB}}$ represents the rotation from the Orbiter body to the Navigation Base reference frame and is a vehicle specific value.

$\mathrm{Q}_{\mathrm{NB}_{\mathrm{B}} \mathrm{R}}$ represents the rotation from the Navigation base to the IMU roll axis (equivalent to the physical IMU case).

$\mathrm{Q}_{\mathrm{C} \_\mathrm{M} 50}$ represents the rotation from a properly aligned platform to the M50 reference frame. This is the same as the current REFSMMAT for each IMU.

Note that Equation 2 assumes that M50 and the inertial reference frame are identical.

The current position, $Q_{\mathrm{P} \text { M50, }}$ of each IMU as determined using ISS as a reference is calculated with Equation 3 using the conjugate of $\mathrm{Q}_{\mathrm{B}_{-} \mathrm{I}}$ as calculated in Equation 2, the conjugates of $\mathrm{Q}_{\mathrm{ISS} \_\mathrm{B}}$ and $\mathrm{Q}_{\mathrm{M} 50 \_ \text {J2K, }}$ and the calibration of the ODS Offset, $\mathrm{Q}_{\mathrm{B} \_ \text {Iss. }}$.

$$
\mathrm{Q}_{\mathrm{P}_{-} \mathrm{M} 50}=Q_{P_{-} I} \cdot Q_{I_{-} B} \cdot Q_{B_{-} I S S} \cdot Q_{I S S_{-} J 2 K} \cdot Q_{J 2 K_{-} M 50}
$$

Where:

$\mathrm{Q}_{\mathrm{P}_{\mathrm{I}}}$ represents the rotation from the present IMU platform position to an inertial frame that is not M50 due to the IMU misaligment. This is numerically equivalent to the current REFSMMAT. Since the current REFSMMAT represents the rotation of a well aligned IMU platform to the M50 frame it also represents the rotation of a poorly aligned IMU platform to some other inertial frame that is itself rotated from M50 by the same amount as the platform misalignment.

From this point the torquing angles that will return the IMU to the current REFSMMAT using Equation 4:

$$
\mathrm{Q}_{\mathrm{P} \__{C}}=Q_{P_{-} M 50} \cdot Q_{M 50 \_C}
$$

Since the IMUs are also physically skewed with respect to each other, the skewing must be taken into account at this point. During this procedure, one IMU is recovered before the other two. The IMU that is being recovered first will be called "IMU X" and "IMU Y" denotes one of the other IMUs and is called the "reference IMU". Since $Q_{P}$ M50 can be calculated for each IMU (X and Y) the skewing between the IMUs is calculated by ( $\left.\mathrm{Q}_{\mathrm{PX} \text { M50_. }} \mathrm{Q}_{\mathrm{M} 50 \_\mathrm{PY}}\right)$. Now the desired rotation for IMU X to M50, QDX_M50, can be calculated using the conjugate of the result of Equation 4 for IMU X, Q_PX.

$$
\mathrm{Q}_{\mathrm{DX} \_\mathrm{M} 50}=Q_{C_{-} P X} \cdot\left(Q_{P_{X_{-} M 50}} \cdot Q_{M_{50} P Y}\right) \cdot Q_{C_{X_{-} M 50}}
$$

$\mathrm{Q}_{\mathrm{DX} \_M 50}$ can now be uplinked to the Orbiter as the "Desired REFSMMAT" and performing an IMU alignment of IMU X to IMU Y will reference $Q_{D X \_M 50}$ to torque IMU X such that it is in the correct position as defined by the "Current REFSMMAT". However, the last step of an IMU to IMU alignment is set the "Current" REFSMMAT equal to the "Desired". In this case the IMU is being torqued to match the "Current" REFSMMAT and the "Desired" REFSMMAT will not accurately describe the position of the IMU at the end of the alignment. Thus the IMU alignment must be manually terminated when the alignment error of each axis is 0.05 deg prior to the alignment software completing the REFSMMAT transfer.

Recovery of IMU X can be verified by the long term eigen angle computations returning to near normal values. To complete the recovery the original (launch) desired REFSMMATs for all three IMUs are uplinked and the other two IMUs are aligned to IMU X. Finally, IMU X is aligned to another IMU to restore launch skewing.

While attitude reference is being recovered it is highly desirable that ISS be in attitude control of the mated stack. If Orbiter must be in control, the reference IMU should be the same as the IMU that is selected for attitude reference onboard to avoid a high maneuver rate during the alignment.

\section{B. With IMU Matrix Aligns}


The procedure to recover IMU attitude reference from ISS became much easier with the addition of an IMU Matrix alignment capability to the Orbiter flight software. The present position of the recovery IMU X platform as referenced from ISS is known from Equation $3\left(\mathrm{Q}_{\mathrm{P}} \mathrm{M}_{50}\right)$. This value can be simply uplinked to the desired REFSMMAT and IMU $\mathrm{X}$ is matrixed aligned to itself. This sets the current REFSMMAT equal to the desired such that the current REFSMMAT once again describes the orientation of IMU platform X. To complete the recovery the original (launch) desired REFSMMATs for all three IMUs are uplinked and the other two IMUs are aligned to IMU $\mathrm{X}$. Finally, IMU $\mathrm{X}$ is aligned to another IMU to restore launch skewing.

\section{Recovery of ISS Attitude Reference from Shuttle}

During STS-117, which launched June 8, 2007, the Russian TVM and TsVM computers began to fail on Flight Day (FD) 5. On Flight Night (FN) 5 the computers failed again and attempts to recover them were unsuccessful. Significant efforts were made to minimize disturbances on the mated stack so Momentum Management would not be in danger of saturation. During this time, many other contingency plans were prepared.

In addition to the Russian computer failures, two of the US GPS units were already failed from an independent problem and the onboard software at that time did not support independent attitude determination with only two GPS units. The US segment was using RGA data to propagate between attitude updates from the Russian segment, but with the loss of the Russian computers these updates were not available. Thus the US Segment was left with no other option than to propagate the last known attitude with RGA data and it was only a matter of time before the propagation error became too large. To workaround this issue, the contingency procedure to recover Shuttle attitude reference from ISS as described above was reversed in order to provide ISS attitude with Shuttle as a reference.

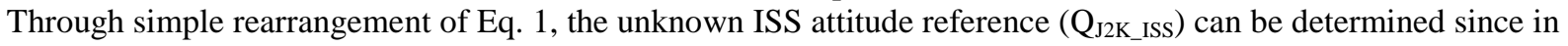
this case all other variables are known utilizing the same "calibrated" ODS Offset (Q___ISS).

By the time all ground calculations were verified, the Russian computers were recovered on FN8. The source of the computer problem was isolated to a common power protection circuit that was able to be bypassed. However to validate the work, Shuttle GNC flight controllers provided the ISS GNC flight controller (ADCO) with the Shuttle derived attitude reference. ADCO used this information to create a command which was uplinked on FD10. The uplinked attitude differed by only 0.35 degrees RSS when compared to the Russian attitude reference.

\section{Direct Deadband Collapse}

When the Russian computers failed for the first time on STS-117, the Orbiter happened to be in attitude control of the mated stack in support of a solar array wing (SAW) deploy. After the required FREE drift period for the deploy, the Orbiter maneuver the mated stack back to the TEA attitude and maintained control during computer troubleshooting. But the question arose: how would Orbiter hand back attitude control to ISS before Orbiter reached critical propellant quantities?

As previously discussed, to preserve the on-board propellant of both vehicles, ISS attitude control is nominally maintained by Momentum Management using the vehicle's four CMGs. When using Shuttle VRCS for mated attitude control worst-case rate errors are expected to be 0.01 to $0.02 \mathrm{deg} / \mathrm{sec}$. Since Momentum Management requires rates of $0.001 \mathrm{deg} / \mathrm{sec}$ or less for startup, nominally the Orbiter would handover attitude control first to USTO, a control mode in which the US segment commands Russian thrusters. This allows the rates to be damped sufficiently for a subsequent handover to Momentum Management. With the computers failed, Russian thrusters were not available. The Russian computers were "temporarily" recovered so that a handover to Momentum Management could be accomplished near the end of FD5, but contingency plans were made in case the computers failed again and could not be recovered. Without Russian thrusters it was unknown if Orbiter VRCS could achieve low enough rates to for Momentum Management start up.

One of the ways initial transients during momentum management startup can be reduced is by ensuring the attitude the mated stack at the time of handover is as close to the attitude momentum management will command as possible.

The Shuttle star trackers provide a quantitative assessment of the accuracy of the Shuttle IMU's and in turn, the Shuttle's attitude reference. Such error quantification on the ISS US segment side is more complicated but can be approximated through comparison with Russian sources. When both vehicles have accurate independent attitude references, the most significant source of error of the relative attitude comparison becomes the ODS offset. In order 
to ensure the attitude the Shuttle commands prior to an attitude control handover is as close to the commanded attitude of Momentum Management, the ISS commanded attitude is transformed into Orbiter coordinates using the ODS offset. This attitude is then loaded into the Shuttle DAP to two decimal places.

Draper Laboratories showed that commanding a rate error limit directly of $0.001 \mathrm{deg} / \mathrm{sec}$ causes stability concerns within the autopilot. Rather than risk DAP stability, the reduced rate error can be achieved by reducing attitude deadbands due to the specific design of the DAP phase plane logic.

During STS-117, a procedure was developed to handover attitude control directly from Orbiter VRCS to ISS momentum management. The procedure utilized two attitude deadband collapses: the first from the nominal 3 deg deadband to $1 \mathrm{deg}$, and then a second collapse down to 0.5 deg. Afterwards FREE drift was commanded and the crew used the Rotational Hand Controller to fly out any rate errors. When the crew was as close to zero rate error as possible, momentum management was started.

Since Russian computers were able to be recovered, this procedure was not executed during STS-117 but was used during STS-118 as a test objective. The procedure was successful during STS-118 and paved the way for future development. Due to the benefits of a direct handover (less ISS propellant used since not firing Russian thrusters, decreased loads on the mated stack since not firing Russian thrusters, and faster achievement of momentum management settlement since ISS does not need to first go through USTO) an operationally easier procedure was desired.

Working along with Draper, the GNC team developed a direct handover procedure that utilizes a single deadband collapse from 3 degrees to $0.1 \mathrm{deg}$. By collapsing all the way down to $0.1 \mathrm{deg}$, manual rate damping is no longer required. This single attitude deadband collapse procedure was first tested on STS-129 and has become the nominal Orbiter to ISS attitude control handover procedure. This improved handover method is only possible when the ODS offset is known and taken into account.

\section{Conclusion}

The Space Shuttle does not dock to the ISS at an exact 90 deg angle as designed. This variation is a significant driver to the accuracy of attitude comparisons between the two vehicles. By comparing the vehicle's attitude references over time, an accurate estimate of the ODS offset can be measured which then allows an attitude reference to be accurately transferred between the two vehicles for contingency scenarios. Knowledge of the true ODS offset angle can be utilized to transfer attitude control from Orbiter to ISS using less ISS propellant, time and decreased loads.

\section{Acknowledgements}

The operational use and measurement of the ODS offset has been refined over the years by the Guidance, Navigation and Control (GNC) Shuttle flight controllers, however, most of the initial work was completed by Charles Alford (ret.) and Eddie Trilca (ret.) in 2003. We would also like to thank Mike Martin and Ed McCants of Draper Laboratories who have assisted our group in the development of the direct attitude control handover procedures. 